\title{
Two white dwarfs with oxygen-rich atmospheres
}

\author{
B.T. Gänsicke ${ }^{1}$, D. Koester ${ }^{2}$, J. Girven ${ }^{1}$, T.R. Marsh ${ }^{1}$, D. Steeghs ${ }^{1}$ \\ ${ }^{1}$ Department of Physics, University of Warwick, Coventry CV4 7AL, UK \\ ${ }^{2}$ Institut für Theoretische Physik und Astrophysik, University of Kiel, 24098 Kiel, Germany
}

\begin{abstract}
Stars with masses in the range $7-10 M_{\odot}$ end their lives either as massive white dwarfs or weak type II supernovae, and there are only limited observational constraints of either channel. Here we report the detection of two white dwarfs with large photospheric oxygen abundances, implying that they are bare oxygen-neon cores and that they may have descended from the most massive progenitors that avoid core-collapse.
\end{abstract}

White dwarfs represent the endpoints of stellar evolution for the overwhelming majority of all stars. Most white dwarfs in the Galaxy have carbon-oxygen (CO) core compositions, being descendants of low to intermediate mass stars that underwent hydrogen and helium core burning. Stars with initial masses $7 M_{\odot} \leq M \leq 10 M_{\odot}$ will reach sufficiently high core temperatures to proceed to carbon burning, and produce either oxygen-neon $(\mathrm{ONe})$ core white dwarfs, or undergo a core-collapse supernova (SN II) via electron capture on the products of carbon burning $(1)$. The exact outcome of stellar evolution in this mass range depends critically on the detailed understanding of mass loss (2), the relevant nuclear reaction rates and the efficiency of convective mixing in the stellar cores (3). Some observational constraints on stellar models come from analysis of SN II progenitors (4), which suggest a lower limit on the progenitor masses of $8_{-1.5}^{+1.0} M_{\odot}$. A linear extrapolation of the observed relation between the masses of white dwarfs and their progenitors (5) up to the maximum mass of white dwarfs leads to a broadly 
consistent result.

Information about the core compositions of white dwarfs, in particular of those descending from the highest possible masses, would help to test and improve the theory of stellar evolution. Unfortunately, almost all white dwarfs have hydrogen and/or helium envelopes that, while low in mass, are sufficiently thick to shield the core from direct view. Asteroseismology has the potential to unveil their core composition (6), but observational studies attempting to exploit this potential remain ambiguous [see e.g. the case of GD 358, (7, 8, 3)]. Core material can be directly detected in the photospheres of a small number of stars (9) that underwent a very late thermal pulse during their asymptotic giant branch (AGB) evolution, ejecting a large fraction of the envelope (10) and leaving a white dwarf with only a thin layer of helium. Examples of hydrogen-deficient post-AGB stars are the hot PG1159 objects, white dwarfs with helium-rich (DB) atmospheres and cool carbon-rich (DQ) white dwarfs. The most extreme case known to date is $1 \mathrm{H} 1504+65$, which is hydrogen and helium deficient, representing a hot, naked $\mathrm{CO}$ surface $(11)$.

Recently, the Sloan Digital Sky Survey (SDSS), through its comprehensive spectroscopic snapshot of the Galactic stellar population, has revealed a small class of hydrogen-deficient white dwarfs (12). Their spectra are consistent with nearly pure carbon atmospheres (13). It has been suggested that these "hot" DQ white dwarfs represent the evolutionary link between objects such as PG1159 and 1H1504+65, the DB white dwarfs, and the cool DQ white dwarfs (14, 15).

Until now, all known white dwarfs for which photospheric oxygen and carbon abundances have been determined have abundance ratios $\mathrm{O} / \mathrm{C} \leq 1$. Some stellar models predict that the most massive stars avoiding core-collapse will result in ONe white dwarfs with very low carbon abundances $(16)$. Should such a core lose its hydrogen envelope, an extremely oxygen-rich spectrum would be expected. Here we present the results of a search for white dwarfs with large 
photospheric oxygen abundances within the SDSS spectroscopic Data Release (DR) 7 (17).

We selected all spectroscopic objects in DR7 that fall within the ugriz color space of the white dwarfs from (18). We then subjected these spectra to an automatic measurement of the equivalent widths [see (19) for details of the method] of the O I $616 \mathrm{~nm}$ and $778 \mathrm{~nm}$ multiplets, which are the strongest O I lines expected in a cool, oxygen-rich atmosphere. Another strong O I multiplet is located at $845 \mathrm{~nm}$, however, this region can be affected by residuals from the night sky line subtraction, and we therefore did not include it in our procedure. As an additional constraint, we required the spectra to have a signal-to-noise ratio $\geq 10$ in the regions around the two O I lines, resulting in the analysis of 25639 SDSS spectra (20).

We found 1000 spectra with a formal $>4 \sigma$ detection of both OI lines, and visual inspection of these spectra showed that 998 were erroneously flagged by our automated routine because of poor data quality. Only two objects with genuine O I $616 \mathrm{~nm}$ and $778 \mathrm{~nm}$ absorption, SDSS J092208.19+292810.9 and SDSS J110239.69+205439.4, survived our scrutiny. Both have high proper motions, $0.275 \% / y r$ and $0.163 \% / y r$, respectively (21), consistent with them being nearby low-luminosity blue stars. Aside from the O I $616 \mathrm{~nm}$ and $778 \mathrm{~nm}$ absorption lines, the spectrum of SDSS $0922+2928$ (Fig. 1) is typical of a cool DQ white dwarf, with strong $\mathrm{C}_{2}$ Swan bands in the blue, superimposed by a number of atomic carbon lines. In contrast SDSS $1102+2054$ is a unique white dwarf (22) with a photospheric spectrum totally dominated by absorption lines of O I (Fig. 1). The only other noticeable features are some weak lines of atomic $\mathrm{C} \mathrm{I}$ as well as $\mathrm{H} \alpha$ and $\mathrm{H} \beta$.

We determined the effective temperatures of the two white dwarfs by fitting their SDSS ugriz photometry with two grids of theoretical atmosphere models: one for a pure He composition [Supporting Online Material (SOM), Sect. 1], and one including H, C, and O as well (SOM, Sect. 2). The white dwarf temperatures and chemical abundances determined from these fits depend only mildly on the assumed surface gravity $\log g$ and, because their masses are not 
constrained by the available observations, we fixed $M=0.6 M_{\odot}$, corresponding to the canonical value of $\log g=8$ [ (23), SOM Sect. 2].

Independent of the details of the atmospheric composition, the photometry suggests that both white dwarfs have temperatures in the range 8000-9000 K (Table 2). The implied distances depend strongly on the assumed surface gravity via the radius, here we used the mass-radius relation of (24). We used the effective temperatures (and assumed surface gravity) to calculate synthetic spectra for a final determination of atmospheric abundances from the absorption lines in the SDSS spectroscopy (SOM, Sect. 2).

For SDSS $1102+2054$, we adopted $T_{\text {eff }}=8150 \mathrm{~K}$ and $\log g=8$ and iterated the abundances of $\mathrm{H}, \mathrm{C}$, and $\mathrm{O}$, until we obtained a reasonable fit to the line strengths. Because this was not possible for all oxygen lines we concentrated on the strong lines at 778 and $845 \mathrm{~nm}$, which originate from the lowest lying levels of all optical lines. These lines depend only weakly on temperature and are best suited for a determination of abundances. The best compromise was achieved for the abundances in Table 3 .

However, for this model the carbon multiplet near $712 \mathrm{~nm}$ and the $834 \mathrm{~nm}$ line, $\mathrm{H} \alpha$, and many OI lines from higher levels are much too broad, indicating too high a pressure in the atmosphere. In addition, O I lines from the higher levels are much too weak. The most likely solution of this discrepancy is to assume a higher temperature, which increases the strength of the high-excitation lines compared to the strong multiplets (SOM, Sect. 2). A reasonable compromise was achieved with $T_{\text {eff }}=10500 \mathrm{~K}, \log g=8$ (Table 3 ). The remaining differences in the absorption lines between the model and observation are likely within the uncertainties of the atomic data and in particular the van der Waals broadening constants (SOM, Sect. 2).

This model, however, is in significant disagreement with the temperature derived from the SDSS photometry. One plausible cause for the discrepancy between the temperatures determined from our fits to the photometry and to the spectroscopy are uncertainties in the ultraviolet 
opacities of an atmosphere of such peculiar composition, which strongly affect the slope of the optical continuum [ (14), SOM Sect. 1].

The atmospheric parameters of SDSS $1102+2054$ thus remain somewhat uncertain. Nevertheless, when varying the parameters within the range demanded by photometry, line strengths and widths, the $\mathrm{C}$ and $\mathrm{O}$ abundances did not change more than factors of $\sim 3$. Thus the $\mathrm{O} / \mathrm{C}$ abundance ratio in SDSS $1102+2054$ is clearly much larger than one, which makes this star unique among the many thousands of known white dwarfs.

Because, according to the photometry, the effective temperature for the two white dwarfs cannot be very different, the carbon abundance in SDSS $0922+2928$ must be higher in order to produce the strong $\mathrm{C}_{2}$ Swan bands. On the other hand, the hydrogen abundance must be lower, because no Balmer lines are visible. We fixed the $\mathrm{H} / \mathrm{He}$ ratio at $10^{-5}$, the upper limit allowed by $\mathrm{H} \beta$. Further reduction did not substantially influence the models because the major electron donors are $\mathrm{C}$ and $\mathrm{O}$. Using $T_{\text {eff }}=8270 \mathrm{~K}$ from the fit to the photometry, and assuming $\log g=8.0$, we obtained a good fit to the spectrum with the oxygen abundance decreased by $\sim 0.2$ dex and a carbon abundance increased by $\sim 0.6$ dex with respect to SDSS $1102+2054$ (Table 3). Therefore, also in SDSS $0922+2928, \mathrm{O} / \mathrm{C}>1$. With the exception of the strong O I multiplets 778 and $845 \mathrm{~nm}$ most of the higher excitation O I lines and the C I lines show the same problem as in SDSS 1102+2054: they are too weak and too broad. This is again much improved at a hotter temperature, e.g. $10000 \mathrm{~K}$, which is, however, in conflict with the photometry (SOM, Sect. 1).

The low H abundance suggests that SDSS 0922+2928 and SDSS 1102+2054, similar as the other classes of hydrogen-deficient white dwarfs, underwent a late shell-flash, leaving a Hedominated atmosphere. At the low temperatures of these stars, the He convection zone extends sufficiently deep to dredge core material up into the atmospheres (25,26). Given the large age of the two stars ( $>500 \mathrm{Myr}$ ), gravitational diffusion will unavoidably lead to a larger concentration 
of carbon in the envelope. The only plausible explanation for the observed O/C abundances is that these two white dwarfs have overall very low carbon mass fractions, and hence represent naked ONe cores. As such, they are distinct from 1H1504+65 and the "hot" DQ white dwarfs.

Most stellar models that produce ONe cores predict a layer of $\mathrm{CO}$ surrounding the core that should be sufficiently thick to avoid upward diffusion of large amounts of oxygen. However, a sequence of stellar evolution calculations approaching the mass boundary of stars forming ONe white dwarfs and those undergoing electron-capture SN II show that the mass of the CO layer decreases with increasing initial stellar mass (27, 28, 16). Thus SDSS 0922+2928 and SDSS 1102+2054 may have descended from the most massive stars avoiding core-collapse, in which case they would be expected to be very massive themselves. Our current data is insufficient to provide any unambiguous measure of the masses of SDSS 0922+2928 and SDSS 1102+2054, however their $\mathrm{C} / \mathrm{He}$ abundance is at the top of the range observed in the carbon-rich sequence of "cool" DQ (23.29) white dwarfs, some of which have parallax measurements implying that they are of high mass.

We explored spectral models for SDSS $1102+2054$ with the abundances kept fixed as in Table 3, but adopting surface gravities of $\log g=8.5$ and 9.0, which correspond to masses of $0.9 M_{\odot}$ and $1.2 M_{\odot}(\mathrm{SOM}$, Sect. 2$)$. Broadly similar fits to the absorption lines can be achieved for higher surface gravities if the temperature is increased by 1000 to $2000 \mathrm{~K}$ as well. For $\log g=9.0$, the strongest O I lines become somewhat too broad compared to the observations, and we conclude that the currently available data is consistent with masses of up to $\sim 1 M_{\odot}$.

Initial models of the evolution of intermediate mass stars predict that ONe cores should also contain significant amounts of magnesium. Updated nuclear reaction rates have lead to a substantial downward revision of the Mg abundances (30). For SDSS 1102+2054, which has the better quality spectrum of the two white dwarfs presented here, we can place an upper limit on the magnesium abundance of $\log [\mathrm{Mg} / \mathrm{He}]<-6.1$ from the absence of the Mg II $448 \mathrm{~nm}$ 
line.

\section{References and Notes}

1. K. Nomoto, ApJ 277, 791 (1984).

2. A. Heger, C. L. Fryer, S. E. Woosley, N. Langer, D. H. Hartmann, ApJ 591, 288 (2003).

3. O. Straniero, I. Domínguez, G. Imbriani, L. Piersanti, ApJ 583, 878 (2003).

4. S. J. Smartt, J. J. Eldridge, R. M. Crockett, J. R. Maund, MNRAS 395, 1409 (2009).

5. K. A. Williams, M. Bolte, D. Koester, ApJ 693, 355 (2009).

6. A. H. Córsico, E. García-Berro, L. G. Althaus, J. Isern, A\&A 427, 923 (2004).

7. T. S. Metcalfe, D. E. Winget, P. Charbonneau, ApJ 557, 1021 (2001).

8. G. Fontaine, P. Brassard, ApJ Lett. 581, L33 (2002).

9. K. Werner, F. Herwig, PASP 118, 183 (2006).

10. F. Herwig, T. Blöcker, N. Langer, T. Driebe, A\&A 349, L5 (1999).

11. K. Werner, T. Rauch, M. A. Barstow, J. W. Kruk, A\&A 421, 1169 (2004).

12. J. Liebert, et al., AJ 126, 2521 (2003).

13. P. Dufour, J. Liebert, G. Fontaine, N. Behara, Nat 450, 522 (2007).

14. P. Dufour, G. Fontaine, J. Liebert, G. D. Schmidt, N. Behara, ApJ 683, 978 (2008).

15. L. G. Althaus, E. García-Berro, A. H. Córsico, M. M. Miller Bertolami, A. D. Romero, ApJ Lett. 693, L23 (2009). 
16. I. J. Iben, C. Ritossa, E. Garcia-Berro, ApJ 489, 772 (1997).

17. K. N. Abazajian, et al., ApJS 182, 543 (2009).

18. D. J. Eisenstein, et al., ApJS 167, 40 (2006).

19. B. T. Gänsicke, T. R. Marsh, J. Southworth, MNRAS 380, L35 (2007).

20. We inspected a representative sub-set of all the DR7 spectra in our sample and estimate that our analysis comprised $\simeq 6000$ genuine white dwarfs. Applying the same color constraints used in our query on DR7 to the DR4 white dwarf catalogue (18) left $\simeq 2400$ objects, which implies that using DR7 more than doubles the available candidate sample.

21. S. Lépine, M. M. Shara, $A J$ 129, 1483 (2005).

22. We specifically did not restrict our search to objects classified as "stars" by the SDSS processing pipeline, as very unusual objects may be mis-identified by the SDSS spectroscopic classification algorithm. Within DR 7, the algorithm correctly identified SDSS 0922+2928 as "star", but left SDSS 1102+2054 as an object of "unknown" type.

23. P. Dufour, P. Bergeron, G. Fontaine, ApJ 627, 404 (2005).

24. M. A. Wood, White Dwarfs, D. Koester, K. Werner, eds., no. 443 in LNP (Springer, Heidelberg, 1995), pp. 41-45.

25. D. Koester, V. Weidemann, E.-M. Zeidler, $A \& A$ 116, 147 (1982).

26. C. Pelletier, G. Fontaine, F. Wesemael, G. Michaud, G. Wegner, ApJ 307, 242 (1986).

27. E. Garcia-Berro, I. Iben, ApJ 434, 306 (1994).

28. E. Garcia-Berro, C. Ritossa, I. J. Iben, ApJ 485, 765 (1997). 
29. D. Koester, S. Knist, $A \& A$ 454, 951 (2006).

30. J. Gutiérrez, R. Canal, E. García-Berro, $A \& A$ 435, 231 (2005).

31. JG has been supported by an STFC studentship. BTG, TRM, and DS have been supported by an STFC rolling grant. We thank D. Townsley for useful discussions. Funding for the SDSS and SDSS-II has been provided by the Alfred P. Sloan Foundation, the Participating Institutions, the National Science Foundation, the U.S. Department of Energy, the National Aeronautics and Space Administration, the Japanese Monbukagakusho, the Max Planck Society, and the Higher Education Funding Council for England. The SDSS Web Site is http://www.sdss.org/. 


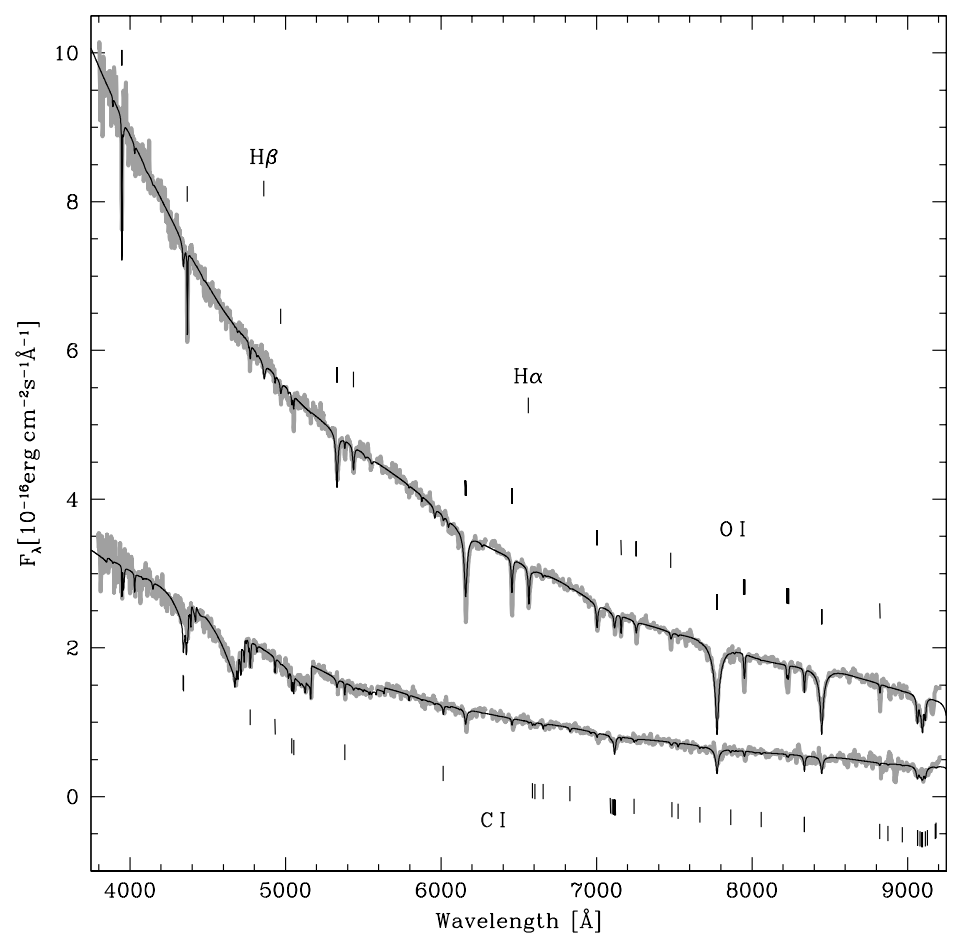

Fig. 1. Sloan Digital Sky Survey spectroscopy. The observed spectra of SDSS 0922+2928 (bottom) and SDSS 1102+2054 (top) are shown as thick gray lines. Superimposed as thin black lines are models for $T_{\text {eff }}=10000 \mathrm{~K}$ and $\log g=8$ (bottom) and $T_{\text {eff }}=10500 \mathrm{~K}$ and $\log g=8$ (top). The wavelengths of the strongest OI and CI absorption lines, as well as of $\mathrm{H} \alpha$ and $\mathrm{H} \beta$ are indicated by tick marks. 
Table 1. Equatorial coordinates, magnitudes and proper motions.

\begin{tabular}{lcc}
\hline & SDSS 0922+2928 & SDSS 1102+2054 \\
\hline RA(2000) & 092208.19 & 110239.69 \\
$\operatorname{Dec}(2000)$ & +292810.9 & +205439.4 \\
$u$ & $18.77 \pm 0.02$ & $17.58 \pm 0.02$ \\
$g$ & $18.52 \pm 0.02$ & $17.24 \pm 0.01$ \\
$r$ & $18.47 \pm 0.02$ & $17.26 \pm 0.01$ \\
$i$ & $18.58 \pm 0.02$ & $17.34 \pm 0.01$ \\
$z$ & $18.71 \pm 0.03$ & $17.46 \pm 0.02$ \\
p.m. & $0.275 " \mathrm{yr}^{-1}$ & $0.163{ }^{\prime} \mathrm{yr}^{-1}$ \\
\hline
\end{tabular}

Table 2. Atmospheric parameters for a surface gravity of $\log g=8$.

\begin{tabular}{lcccc}
\hline System & \multicolumn{2}{c}{$T_{\text {eff }}[\mathrm{K}]$} & \multicolumn{2}{c}{ distance [pc] } \\
& pure He & with metals & pure He & with metals \\
\hline SDSS 0922+2928 & $8720 \pm 260$ & $8270 \pm 320$ & $141 \pm 6$ & $122 \pm 7$ \\
SDSS 1102+2054 & $8820 \pm 110$ & $8150 \pm 150$ & $81 \pm 2$ & $67 \pm 2$ \\
\hline
\end{tabular}

Table 3. Photospheric abundances (by number).

\begin{tabular}{lcccc}
\hline System $\left(T_{\text {eff }} / \log g\right)$ & $\log (\mathrm{H} / \mathrm{He})$ & $\log (\mathrm{C} / \mathrm{He})$ & $\log (\mathrm{O} / \mathrm{He})$ & $\log (\mathrm{O} / \mathrm{C})$ \\
\hline SDSS 1102+2054 (8150/8.0) & -3.2 & -3.2 & -1.8 & 1.4 \\
SDSS 1102+2054 (10500/8.0) & -4.0 & -3.6 & -1.8 & 1.8 \\
SDSS 0922+2928 (8270/8.0) & -5.0 & -2.6 & -2.0 & 0.6 \\
\hline
\end{tabular}




\title{
Supporting Online Material for Two white dwarfs with oxygen-rich atmospheres
}

\author{
B.T. Gänsicke ${ }^{1}$, D. Koester ${ }^{2}$, J. Girven ${ }^{1}$, T.R. Marsh ${ }^{1}$, D. Steeghs ${ }^{1}$ \\ ${ }^{1}$ Department of Physics, University of Warwick, Coventry CV4 7AL, UK \\ ${ }^{2}$ Institut für Theoretische Physik und Astrophysik, University of Kiel, 24098 Kiel, Germany
}

We describe here in more detail the white dwarf model atmospheres that we have computed for the analysis of the SDSS data of SDSS 0922+2928 and SDSS 1102+2054, and outline the approach of our spectral fits. Two grids of atmosphere models were calculated. The first grid consisted of pure helium models, used to provide a first estimate of the effective temperatures of both white dwarfs from fitting their SDSS photometry. The second grid, including $\mathrm{H}$, $\mathrm{C}$, and $\mathrm{O}$, was used to refine the temperature estimate and to determine the atmospheric abundances from fitting the observed absorption lines in the SDSS spectra.

\section{He-model fits to the SDSS photometry}

In order to determine a first estimate of the effective temperatures of the two white dwarfs, we fitted the He-models to the observed ugriz magnitudes, which were corrected to the AB scale by using the corrections suggested by the SDSS project $(-0.04,0.00,0.00,0.01,0.02) \frac{1}{1}$. Because the mass of the two white dwarfs is currently unconstrained by observations, we fixed the surface gravity to the canonical value of $\log g=8.0$. Good fits were achieved for both objects, implying temperatures in the range $8000-9000 \mathrm{~K}$ (Table 2, Fig. S1). We note that for both objects the SDSS data exhibits a discrepancy between the $u$-band flux implied by the photometry, and the extrapolation of the flux-calibrated spectroscopy. As part of our search for white dwarfs with oxygen-rich white dwarfs, we have inspected the SDSS data of a large number of objects, and find that such a discrepancy is rare, but not truly exceptional, and based on the available data, it is not possible to give preference to either the photometry or spectroscopy. Taking the two spectral energy disributions at face value, a fit to the photometry is bound to result in a lower temperature compared to a fit to the spectroscopy. We will come back to this point below.

\footnotetext{
${ }^{1}$ http://www.sdss.org/dr7/algorithms/fluxcal.html\#sdss2ab
} 


\section{Fitting the SDSS spectroscopy with variable $\mathrm{H}, \mathrm{C}$, and $\mathrm{O}$ abundances}

Since $\mathrm{He}$ is practically neutral at the temperatures estimated above, the three other elements $(\mathrm{H}, \mathrm{C}, \mathrm{O})$ provide a significant electron density, which correspondingly changes the opacity and thermal structure of the atmospheres. The equation of state included 6 diatomic molecules $\left(\mathrm{H}_{2}, \mathrm{CH}, \mathrm{OH}, \mathrm{C}_{2}, \mathrm{CO}, \mathrm{O}_{2}\right)$. In particular the very stable molecule $\mathrm{CO}$ is important, because it reduces the atomic carbon abundance considerably. Also included in the second grid was the continuous absorption of $\mathrm{C}$ and $\mathrm{O}$ using cross sections obtained from the TOPBASE database of the Opacity Project (1,2). The necessary O I and CI line data were obtained from the VALD database (3) and from the Kurucz list (CDROM23). Many of the OI lines from Kurucz list that were prominent in the model were not visible or much weaker in the observed spectra, indicating that some of this line data is wrong. We therefore gave preference to the VALD data, where the problematic lines from the Kurucz list are either absent, or have $g f$ values several orders of magnitude smaller. Recent calculations (4) confirm the data for atomic oxygen in the databases we used.

There are a number of OI lines where the core electrons are not in the ground state ${ }^{4} S^{\circ}$ configuration, but in the ${ }^{2} D^{\circ}$ or ${ }^{2} P^{\circ}$ state. In these cases the core electrons (without the excited electron which makes the transition) are not in the configuration of the O II ground state and the ionization therefore would occur to an excited level of the OII ion. This has to be taken into account in our approximate calculation of the van der Waals broadening constants, which depend on the distance of the levels to the ionization limit. We use the ionization limit of that series, that is 16.942 or $18.636 \mathrm{eV}$ instead of the normal $13.618 \mathrm{eV}$. From that limit and the term levels we estimate the broadening constant using standard approximations (5). Similar approximations are also used for the Stark broadening constants.

We re-fitted the SDSS ugriz photometry with $\mathrm{H}, \mathrm{C}$, and $\mathrm{O}$ abundances close to the final values, which resulted in slightly lower temperatures for the two stars compared to the pure He-models (Table 2). In a final step, we varied the abundances of $\mathrm{H}, \mathrm{C}$, and $\mathrm{O}$ while keeping the effective temperatures fixed ( $8270 \mathrm{~K}$ for SDSS $0928+2928$ and $8150 \mathrm{~K}$ for SDSS $1102+2054$ ) and assuming $\log g=8.0$. In this process, we focussed on the strong $\mathrm{OI}$ lines at 778 and $845 \mathrm{~nm}$, which originate from the lowest lying levels of all optical lines and which only mildly depend on the prevailing effective temperature. It turned out that no abundance pattern would provide a fully satisfying fit to all observed lines, and we consider the abundances listed in Table 3 to represent the best compromise.

A specific problem with this model is that the OI lines from the higher levels are much too weak. One way to reduce the pressure in the atmosphere models is to reduce the amount of He. We have calculated a series of models between 7000 and $11000 \mathrm{~K}$ with the $\mathrm{O} / \mathrm{He}$ ratio increased 
up to values $>10$. In all cases the O I lines are much too strong, and we rule out this possibility. The most likely solution to this problem is then to assume a higher temperature, which increases the strength of the high-excitation lines compared to the strong multiplets. Assuming, as before, a canonical $\log g=8$, a reasonably good fit to the absorption lines was achieved with $T_{\text {eff }}=10500 \mathrm{~K}$ (Fig. 1). However, the slope of this model is substantially bluer than the SDSS ugriz photometry (Fig. S1). A fundamental uncertainty in modelling white dwarf spectra with such unusual abundances is our limited knowledge on the sources of ultraviolet opacity in their atmospheres. Any flux absorbed at ultraviolet wavelengths will be re-distributed to longer wavelengths, and hence result in a steepening of the slope in the optical bandpass. An example of the marked differences in the amount of ultraviolet opacity between pure $\mathrm{He}$ and $\mathrm{C}$ atmospheres has been illustrated by (6). Our models predict strong ultraviolet absorption edges of OI and C I (which we include with the values obtained from TOPBASE), and a strong L $\alpha$ line broadened mostly by neutral helium (which we describe with the theory developed by our group). The currently available data does not provide quantative constraints on the ultraviolet characteristics of SDSS $0922+2928$ and SDSS 1102+2054, and hence a systematic uncertainty on their effective temperatures remains. We conclude that, within the framework of the spectroscopic analysis, it is reasonable to prefer the spectroscopic solution of the higher effective temperatures, as it is impossible to satisfactorily model the observed ratio of O I lines with high and intermediate excitation levels at the low (photometric) temperature.

As discussed in the main manuscript, the large $\mathrm{O} / \mathrm{C}$ abundance ratios found for these two white dwarfs strongly suggests that they represent bare oxygen-neon cores that may have descended from massive progenitor stars. Under this assumption, their masses based on any of the current initial mass-final mass relations (7, 8, 9, $10,11,12)$ are expected to be $\sim 1 M_{\odot}$, corresponding to a likely range for the surface gravity of $8.5 \leq \log g \leq 9.0$. In order to explore the effect of a higher gravity on the spectral fit, we have calculated a grid of models spanning the range $\log g=8.0$ to 9.0 . Since the spectrum is largely dominated by the ionization balance of He and the other elements, a change of $\log g$ can to some extent be compensated by a change in temperature, and to first order the abundances do not change significantly. In fact, we do find that broadly similar fits can be achieved when increasing $T_{\text {eff }}$ along with $\log g$, but keeping the abundances fixed at values given in Table 3. Figure S2 illustrates the fits with our canonical model $\left(T_{\text {eff }}=10500 \mathrm{~K}, \log g=8.0\right)$, and models for higher surface gravities, $\left(T_{\text {eff }}=11500 \mathrm{~K}\right.$, $\log g=8.5)$ and $\left(T_{\text {eff }}=12500 \mathrm{~K}, \log g=9.0\right)$. For $\log g=9.0$, the strongest O I lines become somewhat too broad compared to the observations. We conclude that the currently available data are consistent with a broad range of white dwarf masses, including relatively massive $\left(\sim 1 M_{\odot}\right)$ stars, and that the main conclusion of a large $\mathrm{O} / \mathrm{C}$ abundance ratio is independent of the mass assumed for the spectral fitting. 


\section{References and Notes}

1. W. Cunto, C. Mendoza, F. Ochsenbein, C. J. Zeippen, A\&A 275, L5 (1993).

2. N. R. Badnell, et al., MNRAS 360, 458 (2005).

3. N. E. Piskunov, F. Kupka, T. A. Ryabchikova, W. W. Weiss, C. S. Jeffery, A\&AS 112, 525 (1995).

4. G. Ćelik, S. Ateş, European Physical Journal D 44, 433 (2007).

5. A. Unsoeld, Physik der Sternatmosphären (Springer, Berlin, 1955).

6. P. Dufour, G. Fontaine, J. Liebert, G. D. Schmidt, N. Behara, ApJ 683, 978 (2008).

7. S. Catalán, J. Isern, E. García-Berro, I. Ribas, MNRAS 387, 1693 (2008).

8. J. S. Kalirai, et al., ApJ 676, 594 (2008).

9. M. Salaris, A. Serenelli, A. Weiss, M. Miller Bertolami, ApJ 692, 1013 (2009).

10. S. L. Casewell, et al., MNRAS 395, 1795 (2009).

11. P. D. Dobbie, et al., MNRAS 395, 2248 (2009).

12. K. A. Williams, M. Bolte, D. Koester, ApJ 693, 355 (2009). 


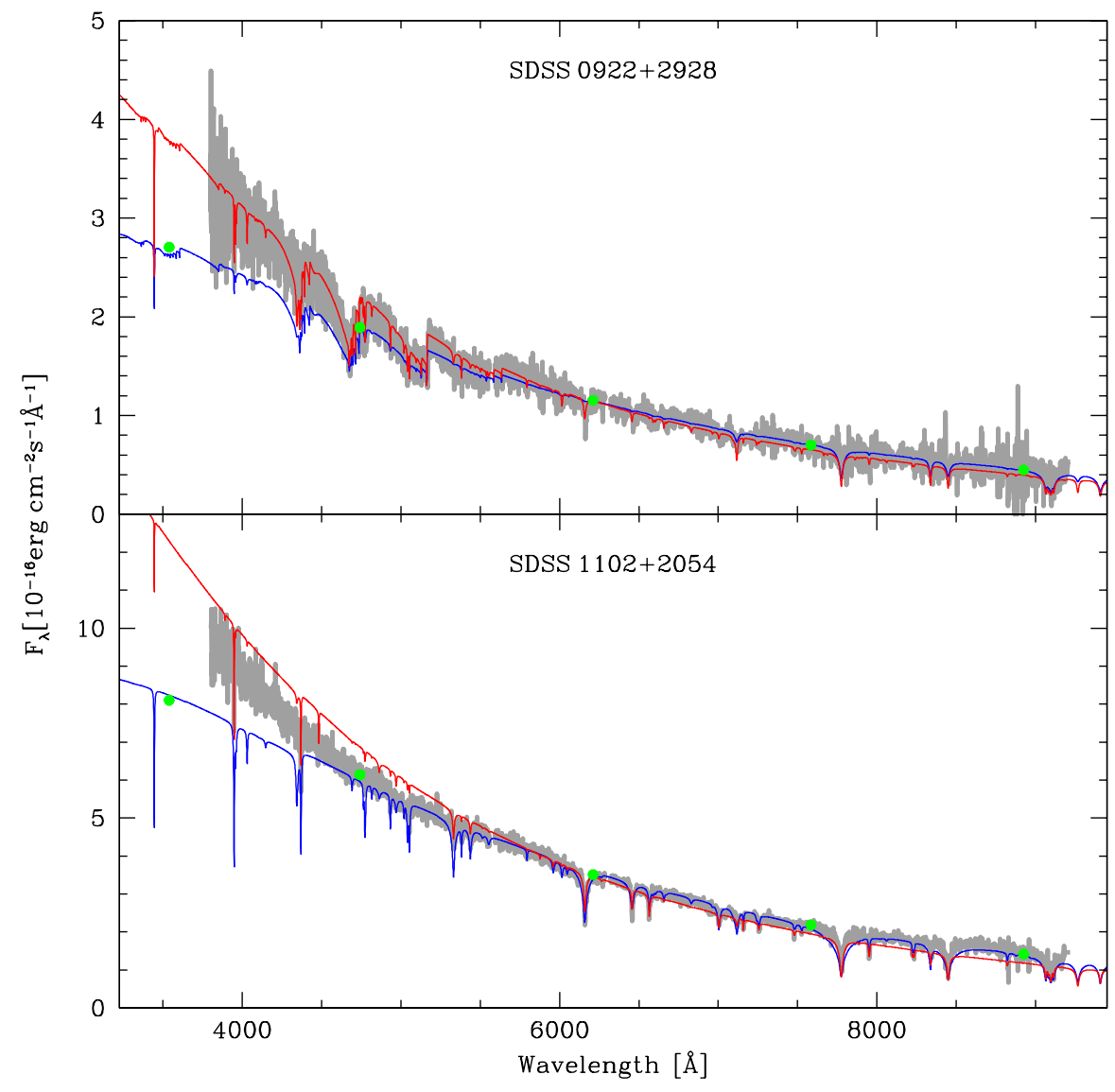

Supporting Figure 1. SDSS spectroscopy (gray lines) and photometry (green dots) of SDSS 1102 +2054 (bottom panel) and SDSS 0922+2928 (top panel). The uncertainties in the fluxes from the SDSS photometry are comparable to the size of the dots. In both cases, the $u$-band flux from the photometry lies below the extrapolation of the spectroscopy. The best-fit models to the photometry for an assumed $\log g=8.0$ are shown as blue lines $\left(T_{\text {eff }}=8270 \mathrm{~K}\right.$ for SDSS $0922+2928$ and $T_{\text {eff }}=8150 \mathrm{~K}$ for SDSS $1102+2054$; see Tables 2 and 3). Shown as red lines are the best-fit models to the absorption lines for an assumed $\log g=8.0\left(T_{\text {eff }}=10000 \mathrm{~K}\right.$ for SDSS $0922+2928$ and $T_{\text {eff }}=10500 \mathrm{~K}$ for SDSS $1102+2054$ ). As part of the fitting the absorption lines, the models were normalised to the observed SDSS spectra. Here, we show the best-fit models scaled to the $r$-band magnitude without that normalisation to illustrate the systematic uncertainty in the optical slope/effective temperature. 

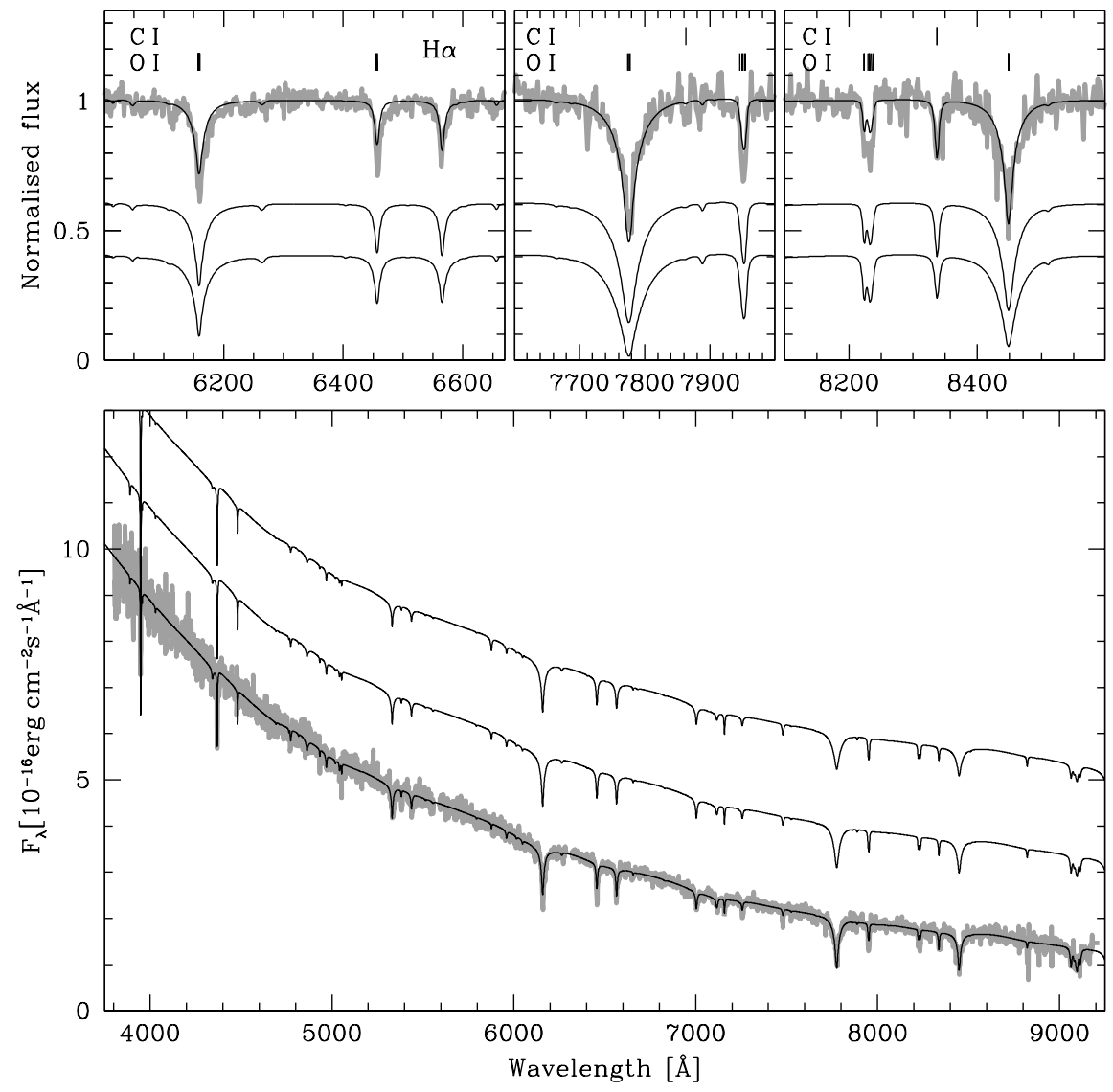

Supporting Figure 2. Model fits to the observed absorption lines of SDSS 1102+2054, illustrating the effect of varying the surface gravity. The central panel shows the full wavelength range of the SDSS spectroscopy, with a model for ( $\left.T_{\text {eff }}=10500 \mathrm{~K}, \log g=8.0\right)$ and the corresponding abundances from Table 3 superimposed on the SDSS data. Note that the continuum slope is normalised to the observed data, and that only the absorption lines are fitted. Offset by two and four flux units are spectra with ( $\left.T_{\text {eff }}=11500 \mathrm{~K}, \log g=8.5\right)$ and $\left(T_{\text {eff }}=12500 \mathrm{~K}, \log g=9.0\right)$, respectively, keeping the abundances fixed to those given in Table 3. The top panels show close-ups of the normalised SDSS spectrum around several O I and C I lines, as well as of $\mathrm{H} \alpha$. Line identifications are indicated by tickmarks. A model for $\left(T_{\text {eff }}=10500 \mathrm{~K}, \log g=8.0\right)$ is superimposed on the observed spectrum, models with ( $\left.T_{\text {eff }}=11500 \mathrm{~K}, \log g=8.5\right)$ and ( $\left.T_{\text {eff }}=12500 \mathrm{~K}, \log g=9.0\right)$ are offset by -0.4 and -0.6 flux units, respectively. 